\title{
EL ACENTO LATINO SEGÚN LOS ANTIGUOS
}

An analysis of the ancient testimonies concerning the Latin accent reveals the meaning of the terminology used by the ancient Roman authors. The absence of accent, non accent, was called "grave». "Circumflex" was used to mean the accent located on the first mora of a long vowel (falling accent). "Acute" had three meanings: 1) any kind of accent, 2) the accent on a short vowel, 3) the accent on the second mora of a long vowel (level accent).

\section{Locus desperatissimus}

Por diversas razones, el acento constituye, en acertada expresión de Liénard ', un locus desperatus de la lingüística latina. Nuestro propósito es examinar uno de los puntos más conflictivos de la cuestión: el testimonio de los antiguos. Es sabido que estos testimonios son el argumento principal en favor de su naturaleza musical. En realidad, el argumento es doble: los antiguos describen el acento latino en los mismos términos que el acento griego, es así que el acento griego era, para casi todos, de índole musical, luego el latino también lo será.

Cuestión previa, pues, es definir a qué tipo de acento podemos dar el calificativo de «musical», y, en segundo lugar, ver si ese concepto es aplicable al griego y, posteriormente, al latín.

\section{Acento intensivo y acento musical}

La primera cuestión es sumamente compleja. Los hodiernos fonetistas, que cuentan con la ventaja de la utilización de los más modernos aparatos, no han podido llegar a un acuerdo sobre el acento musical. Dentro de las lenguas indoeuropeas actuales, tradicionalmente sueco,

' E. Liènard, «Réflexions sur l'accent latin», Hommages à M. Renard, Bruselas 1969, I, p. 560. 
serbocroata y lituano son descritos como lenguas con acento musical. Pero por razones bien distintas. Así, por ej., lenguas consideradas tradicionalmente de acento dinámico, registran modificaciones de altura muy superiores, por ej., a las del serbocroata. Dice Martinet: «Durante mucho tiempo el acento de la mayor parte de las lenguas de Europa se ha considerado como dinámico, es decir, caracterizado por una cima de la curva de la intensidad articulatoria. La observación contemporánea parece indicar que en una lengua como el inglés, por ejemplo, la característica permanente de todo acento es una rápida variación de la curva melódica» ${ }^{2}$. Incluso puede decirse desde los trabajos de Contreras ${ }^{3}$ que es el tono el elemento imperante en el acento del español, desempeñando la intensidad un papel aún más secundario que el de la duración.

La ciencia fonética del s. XIX oponía ambos tipos de acento, tónico e intensivo, como si se tratara de dos realidades químicamente puras e irrenconciliables. La fonética acústica de nuestra época ha hecho ver la exageración de tal postura. Intensidad y altura no sólo pueden combinarse para caracterizar el acento de palabra, sino que éste es el caso más frecuente. Autores como Garde, Gili Gaya, Kurylowicz, Malmberg o Martínez Celdrán ${ }^{4}$, por citar sólo algunos, han subrayado el caduco carácter de tal aséptica contraposición.

\section{Grave, agudo y circunflejo}

Para ser más breve comenzaré por el final. ¿Qué significa entonces el "grave», "agudo" y "circunflejo» de los antiguos? Unánimemente se acepta, pues la interpretación es diáfana, que "grave» no significa otra cosa que "átono»; muchos también ven en "agudo» el término opuesto a "grave" y que caracterizaría, pues, todo acento en general. Es en los dos detalles que siguen donde mi interpretación diverge: yo entiendo que "circunflejo" alude al acento que recae en la primera mora de una vocal larga; y, «agudo", junto al valor indicado, aludiría, como término opuesto a circunflejo, al que recae en la segunda mora. Me parece que esta descripción da perfecta cuenta, hasta donde he podido controlar,

\footnotetext{
${ }^{2}$ A. Martinet, Elementos de lingüistica general, trad. J. Calonge, Madrid 1974, p. 113.

H. Contreras, «Sobre el acento en español», BFUCh 15, 1963, pp. 223-37, y "¿Tiene el español un acento de intensidad?», BFUCh 16, 1964, pp. 237-9. V. también A. Quilis, Fonética acústica de la lengua española, Madrid 1981, p. 327 ss.

${ }^{4}$ P. Garde, L'accent, París 1968, p. 53; S. Gili Gaya, Elementos de fonética general, Madrid $1978_{5}$, p. 32 ss.; J. Kurylowicz, "Akcent i iloczas jako czynnik ritmu", Studia jezykoznawcze, Varsovia 1986, p. 236; B. Malmberg, Les domaines de la phonétique, París 1971, p. 191; E. Martínez Celdrán, Fonética, Barcelona 1984, p. 256 s.
} 
de todos los testimonios antiguos. Es decir, "grave» significa en los textos «no acentuado". "Circunflejo", acento en la primera mora de una vocal larga y sólo ahi, podemos, pues, llamarlo "descendente». "Agudo", "acento en general», incluyendo al circunflejo, pues, y a todo acento sobre vocal breve, así como al específico acento «agudo", que sería aquel que, por oposición al circunflejo, podríamos llamar «ascendente», esto es, sobre la segunda mora de una vocal larga.

Creo, por lo demás, injusto presuponer que los romanos, y, en especial, personas tan sensibles a la lengua como Cicerón o Quintiliano, fueron puros leños para percibir la diferencia natural entre el acento griego y el latino. Los romanos utilizaron, sí, la terminología griega, pero no aplicaron pedisecuamente las normas griegas a su lengua. De entre los claros testimonios de ese no servilismo (Quint., Inst. I 5, 29 s.; Pomp: $G L K$ V 126 s.; Serv. GLK IV 426) selecciono uno expresivo. Ocupándose del acento dice Pompeyo ( $G L K$ V 130): uides, quanta breuitate utantur Latini. Graeci uero chaos fecerunt, totum confuderunt, ut, quamuis mille legas tractatus, non te conuenias. Pero todas las divergencias que los romanos señalan respecto al acento griego hacen referencia a la posición, no a la naturaleza del acento, por lo que hay que suponer que, o todos eran sordos o el acento era sustancialmente de idéntica naturaleza en una y otra lengua, fuera éste ya musical o intensivo.

\section{Etiam pueri sciunt}

Si toda vocal larga estaba - incluso lo niños lo saben-, etiam pueri sciunt, como dice Quintiliano (Inst. IX 4, 47), compuesta de dos breves, de dos moras, de dos tiempos (duorum temporum), como insiste

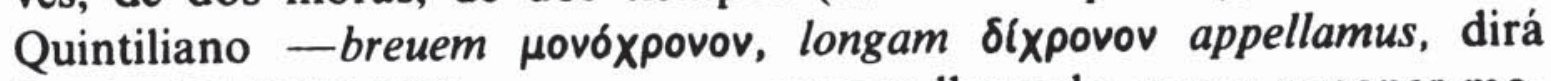
Sergio (GLK IV 533) - o como queramos llamarlo, voy a suponer momentáneamente que, como acaece en todas las lenguas que cuentan con vocales largas, puedo en principio aplicarles para una descripción fonológica el concepto de mora. Puedo suponer las vocales largas latinas, como ya propuso Trubeckoj ${ }^{5}$, como producto de la geminación de una breve y, por tanto, compuestas de dos moras, y que, como suele suceder en estas lenguas, el acento puede recaer en una de esas dos moras.

Unánimemente dicen los antiguos romanos que para disílabos o más el circunflejo se producía sólo cuando la vocal penúltima era larga y la última breve. En todos los demás casos, el acento era agudo.

${ }^{5}$ N. S. Trubeckoj, Principios de fonología, trad. D. Garcia, Madrid 1976 , p. 172. 
Represento ahora la vocal larga como geminada y marco con mayúscula la vocal donde recae el acento según la interpretación que propongo. Así tendríamos un único caso posible para circunflejo hAamus. Pues si la última vocal es larga tendremos siempre acento agudo,

a) ya sea también larga la primera, leEgees,

b) ya sea sólo larga la segunda, pAreens (de pario).

Bien, eventualmente puedo extraer una conclusión de este testimonio. El acento recae en la tercera mora contando desde el final.

Y ¿qué pasa en los otros casos? El acento será, dicen los antiguos, agudo. Así, por ej., cuando las dos últimas vocales sean breves, bOnus, aunque por posición sea larga
a) la primera sílaba, Arma,
b) la segunda sílaba, Arabs,
c) o las dos sílabas, sOllers,

En todos estos ejemplos, las palabras cuentan sólo dos moras. ¿Dónde recae el acento? Sólo y siempre en la segunda, esto es, en lo posible, lo más cerca de la tercera.

¿Y los monosílabos? Si la vocal, dicen los antiguos, es larga, lleva acento circunflejo, en caso contrario, agudo. Tendremos, pues, spEes, pero $p$ Ars o $\mathrm{fEl}$. Es decir, de haber dos moras, otra vez el acento recae en la más cercana a la antepenúltima. Y si, como algunos han defendido, los monosílabos con vocal breve son átonos, entonces el único acento posible en un monosílabo es el circunflejo, sobre la penúltima mora. Lo que es coherente con la ley del alargamiento de los monosílabos autónomos que portan tono. En latín tenemos $t \bar{e}$ con $e$ larga representando, pues, $t E e$ (gr. $\sigma \varepsilon$, dorio $\tau \varepsilon$ ). No hay en latín, según Kurylowicz ${ }^{6}$, por ej., palabras acentuadas del tipo tě con $e$ breve. Aunque sí parece posible que los monosílabos con sílaba larga portaran tono aun cuando su vocal fuese breve $(f E l)$.

¿Puedo deducir entonces que el acento clásico recae siempre que sea posible sobre la mora antepenúltima? Sí, pero con dos restricciones importantes. Una elemental: si sólo se dan dos moras, recaerá en la más cercana a la antepenúltima, es decir, en la penúltima.

¿Y si hay tres o más moras? También. Pero aquí con otra importantísima restricción: en trisílabos o más si la penúltima sílaba es breve, el acento nunca recaerá en la vocal de esa sílaba, sino en la vocal de la sílaba anterior, ya en la tercera mora, fAciunt, ya en la cuarta, fAcioo.

6 J. Kurylowicz, «Metryka a historia języka», Studia... cit., p. 338, y «Réflexions sur l'accent", Problèmes de linguistique indo-européenne, Wroclaw 1977, p. 220. 
Pero nunca más allá de la cuarta, nuUbilis y nuUbilees. Pues expresamente se nos dice (Serv. GLK IV 427, Pomp. GLK V 129) que en sílaba antepenúltima sólo se da el acento agudo y no el circunflejo, es decir, es imposible * $n$ Uubilis o ${ }^{*} n$ Uubilees.

Es precisamente en las condiciones de tal restricción donde el acento latino difiere del de los dialectos eolios. En éstos se impone igual restricción pero condicionada no a la penúltima (vital para el latín), sino

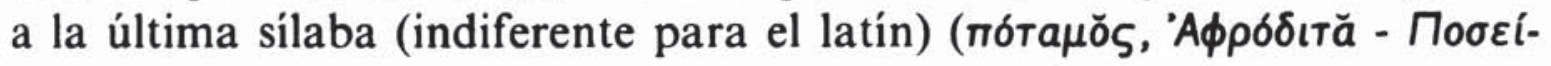
$\delta a ̄ v, \delta$ oúát $\omega)^{?}$.

¿Y si la sílaba penúltima es larga por posición? Entonces ahí necesariamente recaerá el acento, acento agudo, ya en la tercera mora, refEctoos, ya en la segunda, refEctus.

Es decir, el acento recae por orden jerárquico:

1) en la silaba penúltima larga, $y$

2) en la tercera mora. Pero esta situación ideal (hAamus, refEctoos) no siempre se produce.

Cuando la sílaba penúltima no es larga, el acento pasa a la antepenúltima si la hay, ya en la tercera mora ( $f$ Aciunt), ya en la cuarta $(f A$ ciees); y, si no hay antepenúltima, recae en la tercera (IUpoos) o en la segunda mora (IUpus). Cuando no hay sílaba penúltima, lógicamente el acento recae en la última sílaba, pero en la segunda mora ( $s p E e s)$.

Así el acento puede recaer en tres sílabas: penúltima, antepenúltima y última. Así el acento puede recaer sólo en tres moras; tercera, cuarta y segunda. Luego la normativa es ésta:

1) si la silaba penúltima es larga, recaerá en ella el acento, ya en la tercera (CethEegus, refEctoos) o segunda mora (refEctus, CatUllus);

2) si la sílaba penúltima es breve, el acento recaerá en la sílaba antepenúltima, si la hay, ya en la tercera (TUllius), ya en la cuarta mora (nuUbilees); y, si no hay antepenúltima, recaerá en la penúltima, ya en la tercera (IUpoos), ya en la segunda mora (IUpus);

3) si no hay silaba penúltima, por tanto, en monosílabos, el acento recae en la mora más cercana a la tercera, ya en la segunda ( $s p E e s)$, ya necesariamente en la primera $(m E l)$--según los antiguos.

Jerárquicamente, pues, cuando la tercera mora no se encuentra en una sílaba penúltima larga, entonces predomina la sílaba sobre la mora, la sílaba antepenúltima sobre la última, y la cuarta mora sobre la sęgunda.

7 Cf. T. A. Karaseva, "Udarenie v latinskom jazyke i éolijskih dialektah», VMUfilol 4, 1982, p. 58, con tabla comparativa. 


\section{Fonología y Fonética}

Insisto aquí en la posición del acento, porque, como se ha dicho tantas veces, si podemos determinar que es una y sólo una la parte de la palabra que recibe el acento y forzosamente en esa posición un solo tipo de acento, no es fonológicamente relevante si éste se debe a la intensidad, al tono, a la duración o al conjunto de ellos, pues el valor distintivo no corresponde al acento mismo, sino al lugar del acento.

Ahora bien, algo que probablemente nunca lograremos saber con seguridad es si esa acentuación se realizaba como fundamentalmente musical o intensiva, ya total o parcialmente, esto es, sólo para determinadas posiciones del acento, tal como sucede precisamente en serbocroata, cuyos acentos descendentes ("circunflejos») son sobre todo expiratorios y los ascendentes («agudos»), musicales ${ }^{8}$.

Los antiguos definen el circunflejo como un acento compuesto de agudo y grave y lo representan gráficamente (/) como la suma de los signos para el agudo (/) y el grave $(N$ (p. ej., Diom. GLK I 434). Si tomamos al pie de la letra sus testimonios, el circunflejo comportaba una elevación del registro de la voz y una vuelta a su estado inicial. Esto es bastante similar a lo que sucede en serbocroata, lengua, por cierto, como el latín, sin polisílabos oxítonos. En serbocroata la sílaba que sigue al acento ascendente, el agudo, suena bastante alta, mientras que suenan bajas las que siguen al acento descendente o circunflejo. Desde luego, si estipulamos definir el acento serbocroata como musical, entonces el acento latino y el griego deben recibir sin duda igual calificación, pero sin olvidar que es la posición del acento sobre la primera o segunda mora, la llamada correlación de dirección tonal, y no la mayor o menor altura de la voz lo que fonológicamente define su carácter. Sin olvidar, en fin, que la variación de la altura melódica es en serbocroata notablemente menor que en sueco, por ejemplo, y bastante similar (a veces notablemente inferior) tal registro melódico al del ruso o al del eslovaco, lenguas, sin embargo, donde tal fenómeno es puramente alofónico, esto es, no pertinente fonológicamente, al contrario, pues, de lo que sucede en sueco y, en cierta manera, en serbocroata.

\section{Agudo}

Que «agudo» tenga dos acepciones lo prueba este texto de Quintiliano (Inst. I 5, 31): est autem in omni uoce utique acuta, sed numquam plus

${ }^{8}$ N. S. Trubeckoj, o. c., p. 192. 
una... praeterea numquam in eadem flexa et acuta... Ea uero, quae sunt syllabae unius, erunt acutae aut flexa, ne sit aliqua uox sine acuta. Bien, supongamos que "agudo» tenga aquí una sola acepción, no importa cuál.

Quintiliano diría entonces:

a) nunca se da en una misma forma simultáneamente un acento "agudo" y otro circunflejo,

b) toda palabra lleva siempre un «agudo" y sólo uno;

lógicamente, pues, debemos concluir que sólo existe un acento, el «agudo", en cada palabra.

Lo que equivadría a decir que la mente de Quintiliano, Cicerón y todos los demás autores que nos hablan del circunflejo seguia una lógica absolutamente desconocida - por no decir, extraterrestre - para nosotros hablándonos de un acento que no existe, el circunflejo.

Me parece que aquí hay sólo un problema terminológico derivado del doble significado de acutum que alude, por un lado, sí, como muchos han dicho y como se infiere claramente de no pocos testimonios, a todo acento en general en estricta oposición al "grave», $y$, en estricta oposición al circunflejo, a aquel acento situado sobre la segunda mora de una vocal larga.

Con esto, la interpretación del pasaje es diáfana: «toda palabra tendrá un acutum (acento) y sólo uno... en una misma palabra nunca habrá simultáneamente un acento circunflejo (acento descendente) o un acutum (acento ascendente)... Los monosílabos tendrán acento acutum (ascendente) o circunflejo (descendente), de forma que no exista palabra sin acutum (acento)».

Que Cicerón o Quintiliano, dos tratadistas de retórica en los textos que cito, y no dos fonetistas modernos utilizaran una terminología ambigua no es sorprendente. Uno y otro hablan de uox, littera o syllaba sin precisión. A ello hay que sumar, además, el peso de la tradición ter-

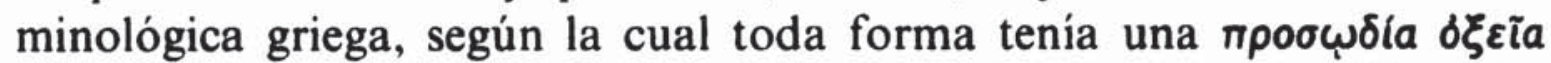

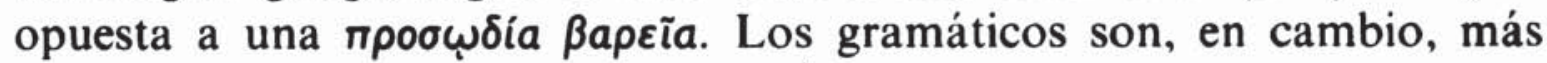
precisos. Por ej., Pompeyo (GLK V 126), quien dice: omnis sermo necesse est ut aut acutum habeat aut circumflexum... si non habet acutum, circumflexum habet; si non habet circumflexum, acutum habet... et grauis ubi erit?... in reliquis syllabis ipsius sermonis.

Por tanto, cuando Cicerón (Orat. 173) habla de "voces agudas y graves" (acutarum grauiumque uocum) está claramente utilizando, como tantas veces se ha dicho, el término acutum por 'acentuado o tónico' y graue por 'no acentuado o átono', y cuando él (Orat. 57) o Quintiliano 
(Inst. I 5, 30 ss.) hablan de acutum y flexum, entonces el primer término vale por 'acento sobre la segunda mora', y flexum por 'acento sobre la primera mora'. En consecuencia, el circunflejo recaerá necesariamente en una vocal larga, reservándose para el acento de toda vocal breve y también para el acento en general el término «agudo».

¿Y por qué se generaliza el término "agudo" para todo acento en general? Dejando al margen la realización fonética precisa - bien coherente en la descripción de los antiguos-, en primer lugar, porque los antiguos (Prisc. GLK III 520; Serg. GLK IV 531) entienden, como vimos, el circunflejo como descomponible en un agudo y en un grave, acutus, grauis et qui ex duobus constat circumflexus, dice Diomedes ( $G L K$ I 431). No hay, pues, sino dos tipos: el agudo y el grave, ya que el circunflejo es la suma de ambos. Se trata, pues, de una ecuación perfecta y legítima, pero, además, estadísticamente - ya lo hemos vistoel circunflejo es infrecuente, en razón de las especiales condiciones prosódicas que exige. Graues numero sunt plures, pauciores acutae, flexae rarissimae, dice Sergio (GLK IV 532).

\section{Circunflejo}

El circunflejo es tradicionalmente el punto más polémico de la acentuación que nos transmiten los gramáticos; puesto que éstos nos dicen - con poderosas razones, como vemos - que el circunflejo «incluye» al agudo, solución socorrida es la de soslayar olímpicamente el circunflejo identificándolo con el agudo. Es un error.

Una acentuación oxítona, excepcional pero explicable, apoya mi interpretación del circunflejo. Expresamente dice Prisciano (GLK II 129 s.), que las formas quae paenultimam habent circumflexam, si patiantur syncopam, seruamus eundem accentum in ultima. Así, todos los ejemplos citados (proodUuc, fuumAat, illic, ArpiinAas) acentúan una sílaba última con vocal larga. Lo que apoya, primero, que el circunflejo sólo se daba en vocal larga $y$, segundo - creo yo-, que se evitaba la mora final.

Dice, además, Diomedes ( $G L K$ I 432) a propósito del tipo ipsius: si uero mediae longae sunt, primae graues, secundae fiunt inflexae (esto es ipsIius, illius) ergo primae acutae sunt, cum media breues (esto es, Ipsius). Obsérvese que aquí se acentúa siempre, por tanto, la tercera mora, y que a ésta, si es la primera de la vocal, se la llama «circunflejo"».

El circunflejo, como dije, nunca recaerá en la sílaba antepenúltima (Serv. GLK IV 427; Pomp. GLK V 129). En efecto, si la vocal es larga 
el acento recaerá necesariamente en la tercera mora o en la más cercana, la cuarta (nuUbilis, nuUbilees), será, pues, ascendente, al que los antiguos llaman "agudo»; y, si cae sobre vocal breve (fAcio) recibe también igual denominación: «agudo».

Pompeyo ( $G L K \mathrm{~V} 126)$ dice que arma no puede llevar sino acento agudo: Arma, y nunca circunflejo, non possumus dicere ârma (esto es, * Aarma). Como dije, el signo $/$ es el normalmente empleado para notar escolásticamente el acento circunflejo. Circumflexus nota de acuto et graui facta, scribitur ita / (Prisc. GLK III 520). Pero para el texto citado por Pompeyo en el aparato crítico de la obra de Keil puede verse una notación excepcional: los mss. $A, B$ y $C$ escriben la vocal geminada (aarma), pero $B$ escribe, además, una $a$ encima de la otra (arma). Es casi el sistema que yo mismo estoy aquí utilizando. Claro, no hay circunflejo sino sobre vocal larga, pues no se trata más que del llamado acento descendente.

Es, por último, esa dicotomía agudo-grave, al menos, la que se impone entre los romanos. Pero existieron otras. Según Sergio tal teoría procedería de Atenodoro, mientras que Dionisio Tracio vería una triple prosodia: aguda, grave y circunfleja; en fin, Tirannión de Amisos habría añadido una cuarta prosodia, la «media», de la que Varrón de Reate fue el portavoz romano. Vt enim inter rudem et eruditum, inter calidum et frigidum, amarum et dulcem, longum et breuem est quiddam medium, quod neutrum est, sic inter imam summaque uocem esse mediam (GLK IV 529). Se trata, pues, de una noción puramente abstracta y teórica, de origen filosófico. Sergio (si es él el autor del libro I de la Explanatio ad Donatum) no sólo no da ejemplo alguno de "acento medio» sino que reconoce las dificultades para percibirlo. Algunos datos que ofrece Sergio son, además, evidentemente singulares respecto a los de los demás gramáticos. Así, aunque la normativa acentual que ofrece es la que ya conocemos, sus fuentes fueron, sin embargo, diversas; por tanto, la uniformidad descriptiva debe provenir no de un tradicional copiarse sucesivamente los unos a los otros - estamos hablando de gramáticos, no de copistas medievales-, sino de unas entidades sustancialmente uniformes e iguales para todos.

\section{Mora y sílaba}

Supuesta la hipótesis de que la realización acentual en griego o latín comportara realmente una variación melódica, si utilizamos como unidad en nuestra descripción la sílaba, entonces ambas lenguas serían po- 
litónicas, pero si utilizamos la mora, ambas serían monotónicas. Precisamente a esta conclusión llega $\mathrm{Hyman}^{9}$ para el griego antiguo, quien señala las dificultades que para muchas descripciones presenta una estricta y aséptica distinción entre lenguas que cuentan moras y lenguas que cuentan sílabas. $Y$ es que, superado el viejo maniqueísmo musicalintensivo, no podemos caer en uno nuevo, el de sílaba-mora. Interesante al respecto es la descripción de la acentuación del beja (lengua sudanesa) de Hudson ${ }^{10}$, éste parte de la clasificación de McCawley ${ }^{11}$, quien propone distinguir de acuerdo a la "unidad de distancia fonológica», esto es, la unidad utilizada para la localización del acento, entre lenguas que cuentan moras («mora-counting») y lenguas que cuentan sílabas («syllable-counting»), y de acuerdo a la "unidad prosódica», esto es, la unidad que de hecho recibe el acento, entre lenguas silábicas («syllable languages») y lenguas mora («mora languages»). Incluso con esta amplia clasificación Hudson observa que la acentuación del beja se ajusta a un tiempo a las cuatro categorías. En el caso del latín, en cambio, éste podría describirse bien como lengua que cuenta sílabas desde el punto de vista de la unidad de distancia fonológica y lengua mora de acuerdo a la unidad prosódica.

Por lo que los antiguos nos dicen, ellos "sentían" su acento como musical, por tanto, gobernado fundamentalmente por la sílaba, por tanto, politónico. Prisciano ( $G L K$ I 7) ejemplificaba los tres tipos de acento con nitidez: árae, àrarum, âra, esto es, aA-rae, aa-rAarum, Aa-ra. Es, pues, significativo su enfoque. Prisciano ejemplifica con la sílaba, y no, como podriamos esperar, con la palabra, áràe-àrârùm-ârà, por ejemplo. Todos estos factores subjetivos son importantes, pues, como afirma Bernardi Perini ${ }^{12}$ : «se è vero che rispeto all'accento ogni lingua o è melodica o è intensiva, è anche vero che tale ripartizione si basa non già sopra un'obiettiva condizione di fatto, bensi sul sentimento che hanno della propria lingua, le singole comunità parlanti».

Que la descripción de la acentuación latina realizada por los antiguos incluya, según mi interpretación, ambos conceptos, el de sílaba y el de la mora, no es sorprendente. Ya antes Trubeckoj, Jakobson o Kurylowicz ${ }^{13}$ hicieron lo propio. No podemos describir la posición del

${ }^{9}$ L. M. Hyman, Fonología. Teoría y análisis, trad. R. Monroy, Madrid 1981, p. 277.

${ }_{10}$ R. A. Hudson, "Syllables, moras and accents in Beja», $J L$ 9, 1973, pp. 53-63.

"J. D. McCawley, The phonological component of a grammar of Japanese, La Haya 1968 , p. 58 ss.

12 G. Bernardi Perini, L'accento latino, Bolonia 1986, p. 2.

13 V. J. P. Kherlakian, "La more, l'équivalence $-=\dot{v}=$ et l'accent latin", Langages 50,1978, p. 38 ss. 
acento con criterios puramente silábicos más que al precio - antifilológico y arbitrario - de condenar al fuego los testimonios de los antiguos.

Que para la localización de la mora acentuada cuente la cantidad vocálica, no la silábica, lo dicen claramente, además, los antiguos. Según Servio (GLK IV 426) in accentibus syllaba siue naturaliter breuis siue positione longa indifferenter accipitur, o como dice Pompeyo (GLK V 130), in accentibus positionem nihil ualere.

Las normas de la acentuación latina que aquí he expuesto no son sino una pura traducción a términos fonológicos modernos de lo que dicen los testimonios antiguos. El resultado de mi descripción no es ni más simple ni más complejo que el dado por otros autores, pero intenta respetar fiel e íntegramente los testimonios antiguos. Y es que me parece enormemente razonable - aunque demasiado prudente - la advertencia final, casi la conclusión del trabajo de Mignot ${ }^{14}$ : "on a peut-être eu tort de récuser le témoignage des grammairiens latins».

Xaverio Ballester

14 X. Mignot, «La place de l'accent en latin», $B S L$ 75, 1980, p. 308. 